\title{
miR-374a-5p inhibits non-small cell lung cancer cell proliferation and migration via targeting NCK1
}

\author{
QIANG GUO $^{1,2}$, HAIJUN WANG $^{3}$, YANZHAO XU $^{1}$, MINGBO WANG $^{1}$ and ZIQIANG TIAN ${ }^{1}$ \\ ${ }^{1}$ Department of Thoracic Surgery, The Fourth Hospital of Hebei Medical University, Shijiazhuang, Hebei 050011; \\ ${ }^{2}$ Department of Thoracic Surgery, Affiliated Hospital of Hebei University, Baoding, Hebei 071000; \\ ${ }^{3}$ Department of Thoracic Surgery, Xingtai People's Hospital, Xingtai, Hebei 054000, P.R. China
}

Received August 2, 2019; Accepted April 16, 2021

DOI: $10.3892 / \mathrm{etm} .2021 .10375$

\begin{abstract}
Emerging studies have indicated that microRNAs (miRNAs/miRs) are involved in regulating non-small cell lung cancer (NSCLC)-associated processes. The present study aimed to evaluate the biological roles of miR-374a-5p in NSCLC. Using reverse transcription-quantitative PCR, the expression levels of miR-374a-5p were determined in NSCLC cells and a normal cell line. Functional experiments were performed to investigate the functions of miR-374a-5p in NSCLC. A luciferase activity reporter assay and rescue experiments were performed to validate NCK adaptor protein 1 (NCK1) as a functional target of miR-374a-5p. It was demonstrated that miR-374a-5p levels were decreased in NSCLC cell lines compared with those in a normal cell line. Furthermore, overexpression of miR-374a-5p inhibited NSCLC cell proliferation and migration in vitro. Of note, NCK1 overexpression reversed the effects of miR-375a-5p on NSCLC cell proliferation and migration. The present results confirmed the tumor suppressor role of miR-374a-5p via targeting NCK1 in NSCLC, indicating the importance of the miR-374a-5p/NCK1 axis in NSCLC.
\end{abstract}

\section{Introduction}

Lung cancer is a leading cause of cancer-associated death worldwide (1). Non-small cell lung cancer (NSCLC) accounts for $80-85 \%$ of all lung cancer cases (2). As the overall survival of patients with NSCLC remains poor, it is essential to identify novel treatment targets for NSCLC.

MicroRNAs (miRNAs/miRs) are a class of non-coding RNAs that lack the ability to code proteins but are capable of regulating gene expression by binding with the 3 '-untranslated

Correspondence to: Dr Ziqiang Tian, Department of Thoracic Surgery, The Fourth Hospital of Hebei Medical University, 12 Jiankang Road, Shijiazhuang, Hebei 050011, P.R. China

E-mail: dr_tianzq@126.com

Key words: microRNA-374a-5p, NCK adaptor protein 1, non-small cell lung cancer, tumor suppressor region (3'-UTR) of target mRNA (3). miRNAs have been reported to serve crucial roles in regulating numerous cellular processes, including proliferation, metastasis and differentiation, and they may have dual roles in carcinogenesis (4-6).

To date, the roles of miR-374a-5p in cancer progression have remained controversial (7-9). A previous study determined that the levels of miR-374a-5p, along with those of miR-195-5p, miR-199a-3p and miR-320a were significantly increased in patients with osteosarcoma (7). Another study revealed that miR-374a-5p was also elevated in patients with triple-negative breast cancer (8). Furthermore, in vitro and in vivo studies demonstrated that miR-374a-5p was able to regulate breast cancer cell proliferation and migration by directly targeting arrestin $\beta 1$ (ARRB1), indicating an oncogenic role of miR-374-5p (8). By contrast, the expression of miR-374a-5p was reduced in esophageal squamous cell carcinoma and negatively regulated by LINC00473 (9). As the significant roles of miR-374a-5p in various cancer types, including NSCLC, have remained elusive, the biological roles of miR-374-5p in NSCLC were herein investigated.

In the present study, the expression of miR-374a-5p was determined in NSCLC cell lines and a normal cell line. The effects of miR-374a-5p on NSCLC cell proliferation and migration were investigated in vitro. A bioinformatics target prediction and a luciferase reporter assay were utilized to identify and confirm the direct binding of miR-374a-5p with NCK adaptor protein 1 (NCK1). The present results may provide novel mechanisms underlying the carcinogenesis of NSCLC and a basis for targeted therapy.

\section{Materials and methods}

Cell culture. The NSCLC cell lines A549 and H1299 and the human bronchial epithelial cell line 16-HBE were purchased from the Cell Bank of the Chinese Academy of Sciences. These cells were incubated in RPMI-1640 medium (Invitrogen; Thermo Fisher Scientific, Inc.) containing $10 \%$ fetal bovine serum (Invitrogen; Thermo Fisher Scientific, Inc.) and 1\% Penicillin-Streptomycin at $37^{\circ} \mathrm{C}$ in a humidified incubator containing $5 \% \mathrm{CO}_{2}$.

Cell transfection. NSCLC cells were transfected with $50 \mathrm{nM}$ miR-374a-5p mimics (cat. no. miR10000727-1-5), negative 
A

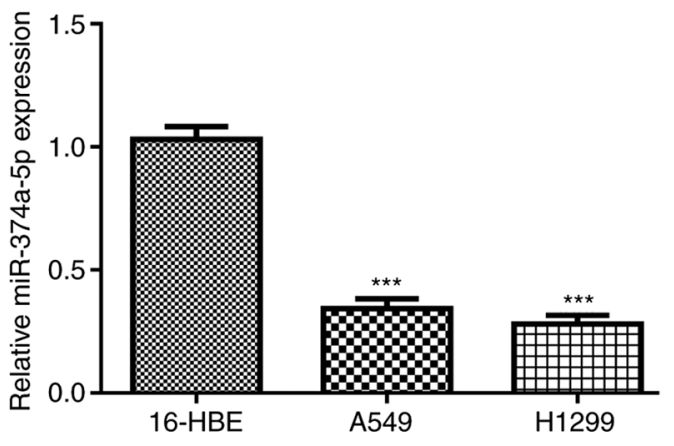

C
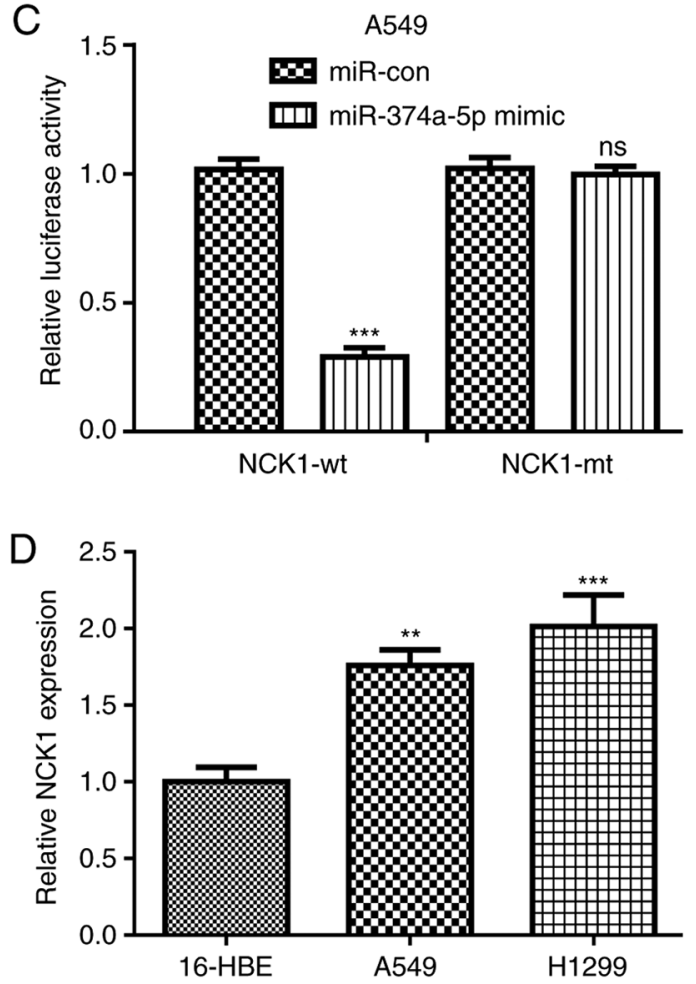

\author{
B NCK1-wt 5'-GACAAUAAGUAUUUUUAUUAUAA-3' \\ | || || || \\ miR-374a-5p 3'-GUGAAUAGUCCAACAUAAUAUU-5' \\ NCK1-mt 5'-GACAAUAAGUAUUUUAUAAUAUA-3'
}
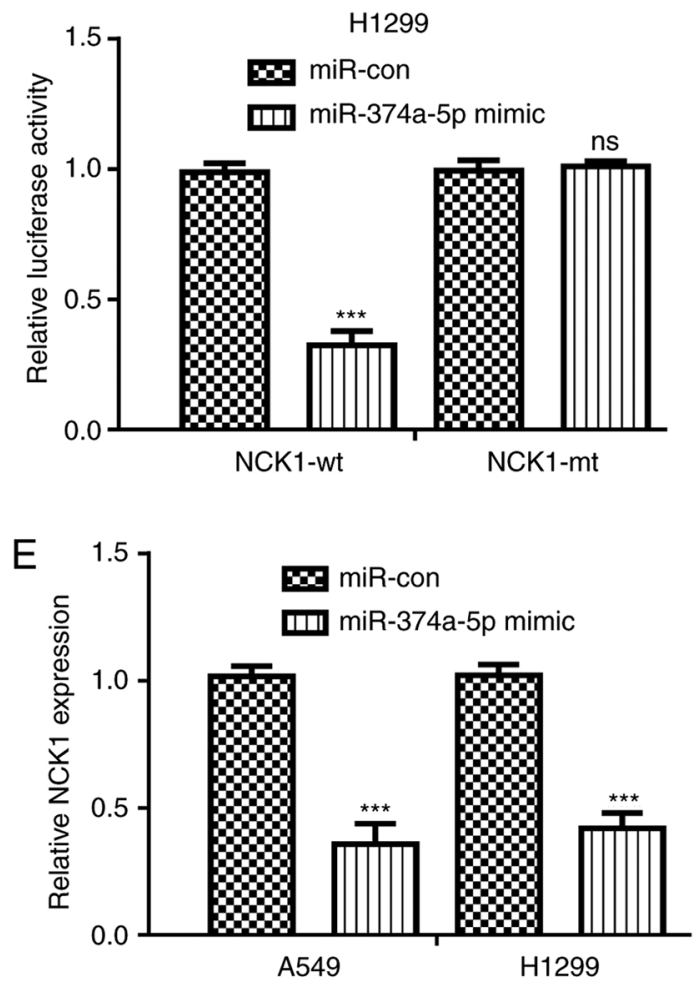

Figure 1. NCK1 is a target of miR-374a-5p in NSCLC. (A) miR-374a-5p expression in NSCLC cell lines (A549 and H1299) and a normal cell line (16-HBE). ${ }^{* * *} \mathrm{P}<0.001$ vs. the 16-HBE group. (B) Sequence alignment indicates the binding region between miR-374a-5p and the 3'-UTR of NCK1. (C) Luciferase activity in NSCLC cells transfected with luciferase activity vectors and synthetic miRNAs. ${ }^{* * *} \mathrm{P}<0.001$ vs. the miR-con group. (D) NCK1 expression in NSCLC cell lines and normal cell line. ${ }^{* *} \mathrm{P}<0.01$ and ${ }^{* * *} \mathrm{P}<0.001$ vs. the miR-con group (E) Effects of miR-374a-5p mimics on NCK1 expression. ${ }^{* * *} \mathrm{P}<0.001$ vs. the miR-con group. NCK1, NCK adaptor protein 1; miR-374a-5p, microRNA-374a-5p; NSCLC, non-small cell lung cancer; miR-con, negative control miR; UTR, untranslated region; wt, wild-type; $\mathrm{mt}$, mutant; ns, no significance.

control miRNA (miR-con; cat. no. miR1N0000001-1-5), $4 \mu \mathrm{g}$ pcDNA3.1 containing the open reading frame of NCK1 (pNCK1) or negative control (pcDNA3.1) purchased from RiboBio with Lipofectamine ${ }^{\circledR} 2000$ (Invitrogen; Thermo Fisher Scientific, Inc.) at $37^{\circ} \mathrm{C}$. After $48 \mathrm{~h}$ transfection, cells were collected for analysis.

Reverse transcription-quantitative PCR (RT-qPCR). RT-qPCR was performed to determine the expression levels of miR-374a-5p or NCK1 in NSCLC cells and the normal cell line. In brief, total RNA from cultured cells was isolated with TRIzol ${ }^{\circledR}$ reagent (Invitrogen; Thermo Fisher Scientific, Inc.). To detect the expression levels of miR-374a-5p, cDNA was synthesized from RNA using the TaqMan MicroRNA Reverse Transcription kit (Applied Biosystems; Thermo Fisher Scientific, Inc.). For the analysis of NCK1 expression levels, the PrimeScript kit (Takara Biotechnology, Co., Ltd.) was used to synthesize cDNA from RNA. RT-qPCR was performed using SYBR Green Mix (Takara Biotechnology, Co., Ltd.) in an ABI 7500 system (Applied Biosystems; Thermo Fisher Scientific, Inc.) with the following thermocycling conditions: $95^{\circ} \mathrm{C}$ for $10 \mathrm{~min}$, followed by 40 cycles of $94^{\circ} \mathrm{C}$ for $45 \mathrm{sec}, 55^{\circ} \mathrm{C}$ for $30 \mathrm{sec}$ and $72^{\circ} \mathrm{C}$ for $1 \mathrm{~min}$. The primers used were as follows: miR-374a-5p forward, $5^{\prime}-\mathrm{GCG}$ CGCTTATAATACAACCTGA-3' and reverse, 5'-GTGCAG GGTCCGAGGT-3'; U6 small nuclear (sn)RNA forward, 5'-CTCGCTTCGGCAGCACA-3' and reverse, 5'-ACGCTT CACGAATTTGCGT-3'; NCK1 forward, 5'-GAACCATCA CCTCCACAGTG-3' and reverse, 5'-AATCCCCTTCAT GTCCTCTTTC-3'; GAPDH forward, 5'-GAAGGTGAA GGTCGGAGTC-3' and reverse, 5'-GAAGATGGTGATGGG ATTTC-3'. Relative expression levels were calculated using the $2^{-\Delta \Delta \mathrm{Cq}}$ method using U6 snRNA or GAPDH as internal controls (10). 

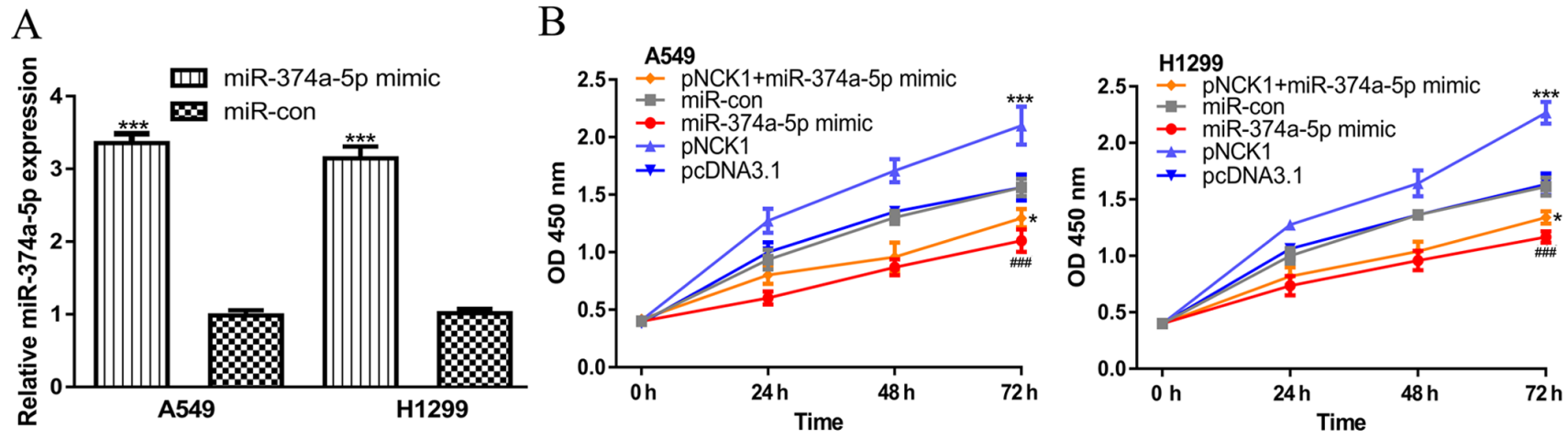

C
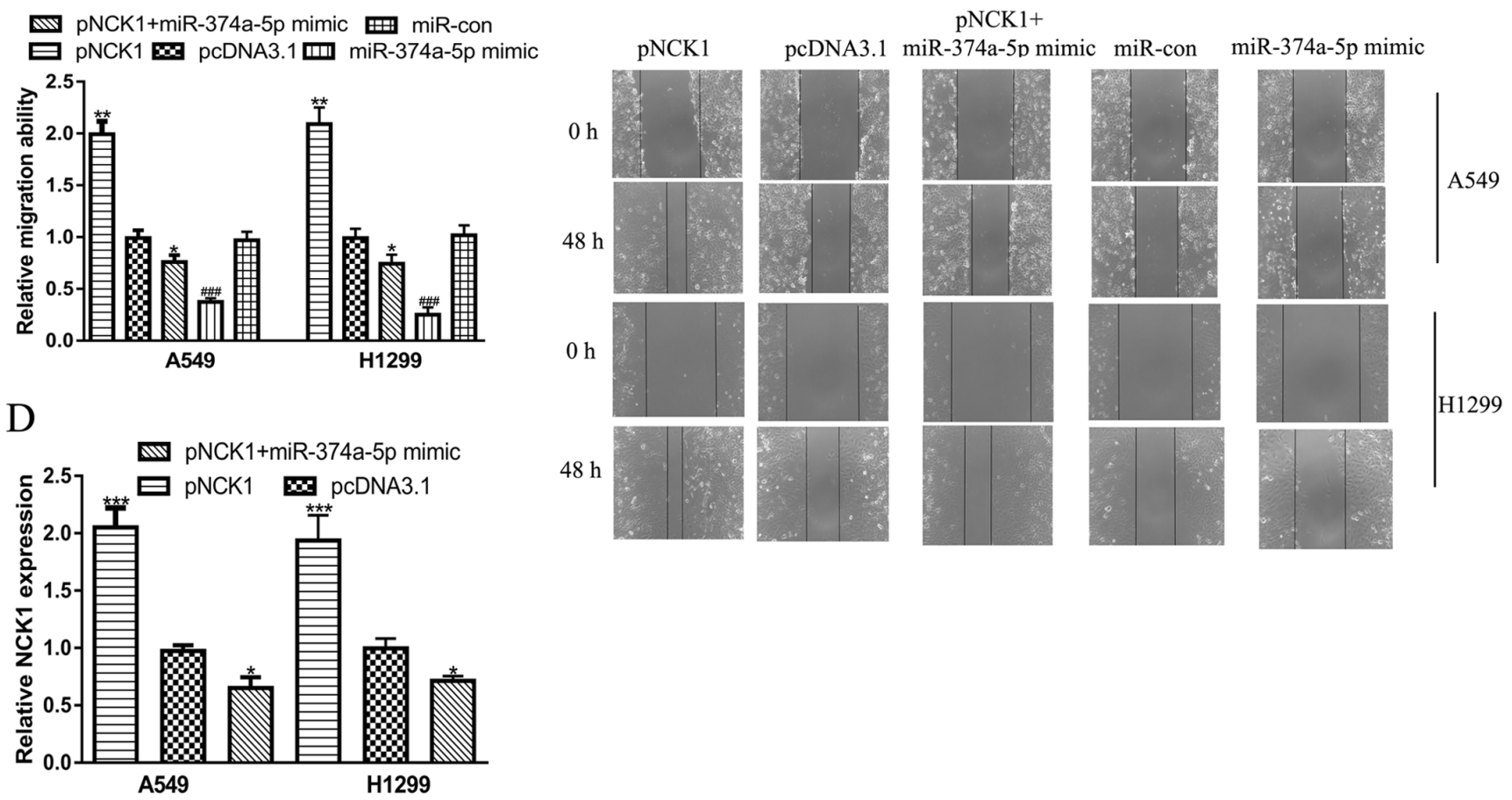

Figure 2. NCK1 reverses the effects of miR-374a-5p on NSCLC cell behaviors. (A) miR-374a-5p expression in NSCLC cells with miR-374a-5p mimics or miR-con transfection. ${ }^{* * *} \mathrm{P}<0.001$ vs. the miR-con group. (B) Cell proliferation was assessed. ${ }^{* * *} \mathrm{P}<0.001$ vs. the pcDNA3.1 group, ${ }^{*} \mathrm{P}<0.05$ vs. the pcDNA3.1 group and ${ }^{\# \# \#} \mathrm{P}<0.001$ vs. the miR-con group. $(\mathrm{C})$ cell migration (magnification, $\mathrm{x} 40$ ) was determined. ${ }^{* *} \mathrm{P}<0.01$ vs. the pcDNA3.1 group, ${ }^{*} \mathrm{P}<0.05$ vs. the pcDNA3.1 group and ${ }^{\# \#} \mathrm{P}<0.001$ vs. the miR-con group. (D) NCK1 expression of NSCLC cells transfected with miR-374a-5p mimics, miR-con, pNCK1, pcDNA3.1 or pNCK1 and miR-374a-5p mimics is presented. ${ }^{* * *} \mathrm{P}<0.001$ and ${ }^{*} \mathrm{P}<0.05$ vs. the pcDNA3.1 group. NCK1, NCK adaptor protein 1 ; pNCK1, NCK1 overexpression plasmid; pcDNA3.1, empty vector; miR-374a-5p, microRNA-374a-5p; NSCLC, non-small cell lung cancer; miR-con, negative control miR; OD, optical density.

Cell proliferation assay. The cell proliferation rate was analyzed with a Cell Counting Kit-8 (CCK-8; Takara Biotechnology, Co., Ltd.). Cells were seeded in 96-well plates at $2 \times 10^{3}$ cells per well and incubated at $37^{\circ} \mathrm{C}$ for $0,24,48$ or $72 \mathrm{~h}$. A total of, $10 \mu \mathrm{l} \mathrm{CCK-8} \mathrm{reagent} \mathrm{(Beyotime} \mathrm{Institute} \mathrm{of}$ Biotechnology) was added to each well, followed by further incubation at $37^{\circ} \mathrm{C}$ for $4 \mathrm{~h}$. The absorbance was measured at $450 \mathrm{~nm}$ using a microplate reader.

Wound healing assay. Serum starved cells were seeded in 6 -well plates at a density of $2 \times 10^{5}$ cells/well and incubated until $100 \%$ confluence was reached. A sterile pipette tip was used to create a wound at the cell surface. The cells were then washed with PBS to remove the cell debris. The migration distance was measured at 0 and $24 \mathrm{~h}$ under a light microscope at a magnification of $\times 200$.
Bioinformatics analysis. Targets for miR-374a-5p were predicted using TargetScan (https://www.targetscan.org) and PicTar (http://pictar.mdc-berlin.de/).

Construction of luciferase activity vectors. According to the results of the bioinformatics prediction, NCK1 was selected as a putative target for miR-374a-5p. The wild-type (wt) 3'-UTR containing the binding site for miR-374a-5p was synthesized by GenScript, cloned into pGL3 vector (Promega Corporation) and named as NCK1-wt. Mutant (mt) 3'-UTR of NCK1 was constructed from NCK1-wt using a site-direct mutagenesis kit (Takara Biotechnology, Co., Ltd.) and named as NCK1-mt.

Dual-luciferase reporter assay. For the luciferase activity assay, NSCLC cells were co-transfected with NCK1-wt or NCK1-mt and miR-374a-5p mimics or miR-con using 
Lipofectamine 2000 with the pGL3 vector described above. After $48 \mathrm{~h}$ of transfection, the cells were lysed to analyze the relative luciferase activities using a dual-luciferase detection kit (Promega Corporation) following the manufacturer's protocol and normalized to Renilla luciferase.

Statistical analysis. GraphPad Prism 5 software (GraphPad Software, Inc.) was used for data analysis. Values are expressed as the mean \pm standard deviation. Differences between groups were analyzed using Student's t-test or one-way analysis of variance with Tukey's post-hoc test. $\mathrm{P}<0.05$ was considered to indicate statistical significance.

\section{Results}

miR-374a-5p is downregulated in NSCLC. First, miR-374a-5p expression levels in NSCLC cells were determined by RT-qPCR analysis. The results indicated that miR-374a-5p expression levels were significantly decreased in NSCLC cells in comparison with those in the normal cell line (Fig. 1A).

NCK1 is a direct target of miR-374a-5p in NSCLC. The bioinformatics tools predicted that NCK1 contains a putative binding site for miR-374a-5p (Fig. 1B). To investigate the interaction of miR-374a-5p and NCK1, luciferase reporter vectors were constructed. The results indicated that miR-374a-5p overexpression decreased the luciferase activity of cells transfected with NCK1-wt, but not of those transfected with NCK1-mt (Fig. 1C). RT-qPCR analysis indicated that NCK1 expression was increased in NSCLC cells compared with that in the normal cell line (Fig. 1D). In addition, NCK1 expression was significantly decreased in NSCLC cells transfected with miR-374a-5p mimics (Fig. 1E).

miR-374a-5p regulates $N S C L C$ cell proliferation and migration via regulating $N C K 1$. The biological roles of miR-374a-5p and NCK1 in NSCLC were investigated (Fig. 2). NSCLC cells were successfully transfected with miR-374a-5p mimics (Fig. 2A). The results of the CCK-8 assay presented in Fig. 2B illustrated that overexpression of miR-374a-5p decreased NSCLC cell proliferation. The wound-healing assay suggested that miR-374a-5p mimics significantly inhibited the migration ability of NSCLC cells (Fig. 2C). To test whether miR-374a-5p regulated NSCLC cell behavior via NCK1, rescue experiments were performed. The overexpression of NCK1 by pNCK1 was confirmed by RT-qPCR (Fig. 2D). The CCK-8 and wound-healing assays indicated that overexpression of NCK1 promoted NSCLC cell proliferation and migration (Fig. 2B and $\mathrm{C}$, respectively). In addition, co-transfection of pNCK1 partially abrogated the effects of miR-374a-5p mimics on NSCLC cell behavior (Fig. 2B and C).

\section{Discussion}

miRNAs may function as either oncogenic or tumor suppressor miRNAs in cancer (11). To date, numerous miRNAs have been indicated to regulate NSCLC progression. Among these, miR-199a-5p was indicated to be markedly decreased in NSCLC tissues (12). In addition, miR-199a-5p overexpression inhibited NSCLC cell proliferation by causing cell cycle arrest at the G1 phase via targeting mitogen-activated kinase kinase kinase 11 (12). Furthermore, miR-221-3p expression was determined to be elevated in NSCLC tissues and cell lines (13). Overexpression of miR-221-3p was also indicated to promote cell cycle progression of NSCLC cells via targeting p27 (13). Increased expression of miR-421 was identified in NSCLC tissues and cells, and its overexpression was able to promote cancer cell migration and invasion through HOP homeobox via the Wnt/ $\beta$-catenin signaling pathway (14).

To the best of our knowledge, the present study was the first to reveal that miR-374a-5p was significantly downregulated in NSCLC cell lines compared with those in a normal cell line. Increased miR-374a-5p expression was also indicated to inhibit NSCLC cell proliferation and migration in vitro. Collectively, the present results suggested a tumor suppressor role of miR-374-5p in NSCLC, which is consistent with its role in esophageal squamous cell carcinoma (9).

miR-374a-5p exerts its effects in cancers by regulating the expression of tumor-specific genes $(8,9)$. For instance, ARRB1 and spindlin 1 were identified as direct targets for miR-374a-5p in different cancer types $(8,9)$. However, the targets of miR-374a-5p in NSCLC remain to be verified. By utilizing TargetScan and PicTar, NCK1 was identified as a putative target for miR-374a-5p. NCK1 is a protein that has been reported to be closely associated with cancer progression $(15,16)$. For instance, NCK1 was determined to be a downstream effector of STAT3 and to promote colorectal cancer cell metastasis and angiogenesis via activating the p21-activated serine-threonine kinase (PAK1)/ERK pathway (15). Furthermore, NCK1 was indicated to promote cervical squamous carcinoma cell angiogenesis via the Rac1/PAK1/matrix metalloproteinase 2 signaling pathway (16). In the present study, the molecular mechanisms of the effects of miR-374a-5p on NSCLC cell behaviors were investigated. Rescue experiments were performed, which demonstrated that NCK1 overexpression partially reversed the effects of miR-374a-5p. These results indicated that miR-374a-5p exerted a tumor-suppressive role through NCK1 in NSCLC. However, as a limitation of the present study, the signaling pathways involved in the regulatory effect of miR-374a-5p on NSCLC cell behaviors were not further investigated.

In conclusion, the present study revealed the reduced expression status of miR-374a-5p in NSCLC and validated that miR-374a-5p functions as a tumor suppressor to regulate NSCLC progression via targeting NCK1. The study provided evidence to unravel a novel miR-374-5p and NCK1 axis in NSCLC, which may help to develop targeted therapies for NSCLC.

\section{Acknowledgements}

Not applicable.

\section{Funding}

This project was supported by Pre-clinical Transformation Research of panel, a Multigene Molecular Marker for Early Diagnosis of Lung Adenocarcinoma ('Office-School Consultation Fund-Science and Technology Innovation' 
Project of Hebei Science and Technology Department-Hebei Medical University; grant. no. 2020TXZH04).

\section{Availability of data and materials}

The datasets used and/or analyzed during the present study are available from the corresponding author on reasonable request.

\section{Authors' contributions}

QG, HW, YX, MW and ZT were involved in designing the study, performed the experiments and interpreted the data. QG and ZT confirmed the authenticity of raw data, and drafted and revised the manuscript. All authors read and approved the final version of the manuscript.

\section{Ethics approval and consent to participate}

Not applicable.

\section{Patient consent for publication}

Not applicable.

\section{Competing interests}

The authors declare that they have no competing interests.

\section{References}

1. Siegel RL, Miller KD and Jemal A: Cancer statistics, 2018. CA Cancer J Clin 68: 7-30, 2018.

2. Bray F, Ferlay J, Soerjomataram I, Siegel RL, Torre LA and Jemal A: Global cancer statistics 2018: GLOBOCAN estimates of incidence and mortality worldwide for 36 cancers in 185 countries. CA Cancer J Clin 68: 394-424, 2018.
3. He L and Hannon GJ: MicroRNAs: Small RNAs with a big role in gene regulation. Nat Rev Genet 5: 522-531, 2004.

4. Bartel DP: MicroRNAs: Genomics, biogenesis, mechanism, and function. Cell 116: 281-297, 2004.

5. Lages E, Ipas H, Guttin A, Nesr H, Berger F and Issartel JP: MicroRNAs: Molecular features and role in cancer. Front Biosci (Landmark Ed) 17: 2508-2540, 2012.

6. Calin GA and Croce CM: MicroRNA signatures in human cancers. Nat Rev Cancer 6: 857-866, 2006.

7. Lian F, Cui Y, Zhou C, Gao K and Wu L: Identification of a plasma four-microRNA panel as potential noninvasive biomarker for osteosarcoma. PLoS One 10: e0121499, 2015.

8. Son D, Kim Y, Lim S, Kang HG, Kim DH, Park JW, Cheong W, Kong HK, Han W, Park WY, et al: miR-374a-5p promotes tumor progression by targeting ARRB1 in triple negative breast cancer. Cancer Lett 454: 224-233, 2019.

9. Chen W, Zhang Y, Wang H, Pan T, Zhang Y and Li C: LINC00473/miR-374a-5p regulates esophageal squamous cell carcinoma via targeting SPIN1 to weaken the effect of radiotherapy. J Cell Biochem 120: 14562-14572, 2019.

10. Livak KJ and Schmittgen TD: Analysis of relative gene expression data using real-time quantitative PCR and the 2(-Delta Delta C(T)) method. Methods 25: 402-408, 2001.

11. Zhang B, Pan X, Cobb GP and Anderson TA: MicroRNAs as oncogenes and tumor suppressors. Dev Biol 302: 1-12, 2007.

12. Li Y, Wang D, Li X, Shao Y, He Y, Yu H and Ma Z: MiR-199a-5p suppresses non-small cell lung cancer via targeting MAP3K11. J Cancer 10: 2472-2479, 2019.

13. Yin G, Zhang B and Li J: miR-221-3p promotes the cell growth of non-small cell lung cancer by targeting p27. Mol Med Rep 20: 604-612, 2019

14. Liang H, Wang C, Gao K, Li J and Jia R: MicroRNA-421 promotes the progression of non-small cell lung cancer by targeting HOPX and regulating the Wnt/ $\beta$-catenin signaling pathway. Mol Med Rep 20: 151-161, 2019.

15. Zhang F, Lu YX, Chen Q, Zou HM, Zhang JM, Hu YH, Li XM, Zhang WJ, Zhang W, Lin C and Li XN: Identification of NCK1 as a novel downstream effector of STAT3 in colorectal cancer metastasis and angiogenesis. Cell Signal 36: 67-78, 2017.

16. Xia P, Huang M, Zhang Y, Xiong X, Yan M, Xiong X, Yu W and Song E: NCK1 promotes the angiogenesis of cervical squamous carcinoma via Rac1/PAK1/MMP2 signal pathway. Gynecol Oncol 152: 387-395, 2019.

This work is licensed under a Creative Commons Attribution-NonCommercial-NoDerivatives 4.0 International (CC BY-NC-ND 4.0) License. 